$12 / 28894850$

UCRL-ID-114780

\title{
Assessment of Ambient-Temperature, High-Resolution Detectors for Nuclear Safeguards Applications
}

Wayne D. Ruhter

James. H. McQuaid

Anthony Lavietes

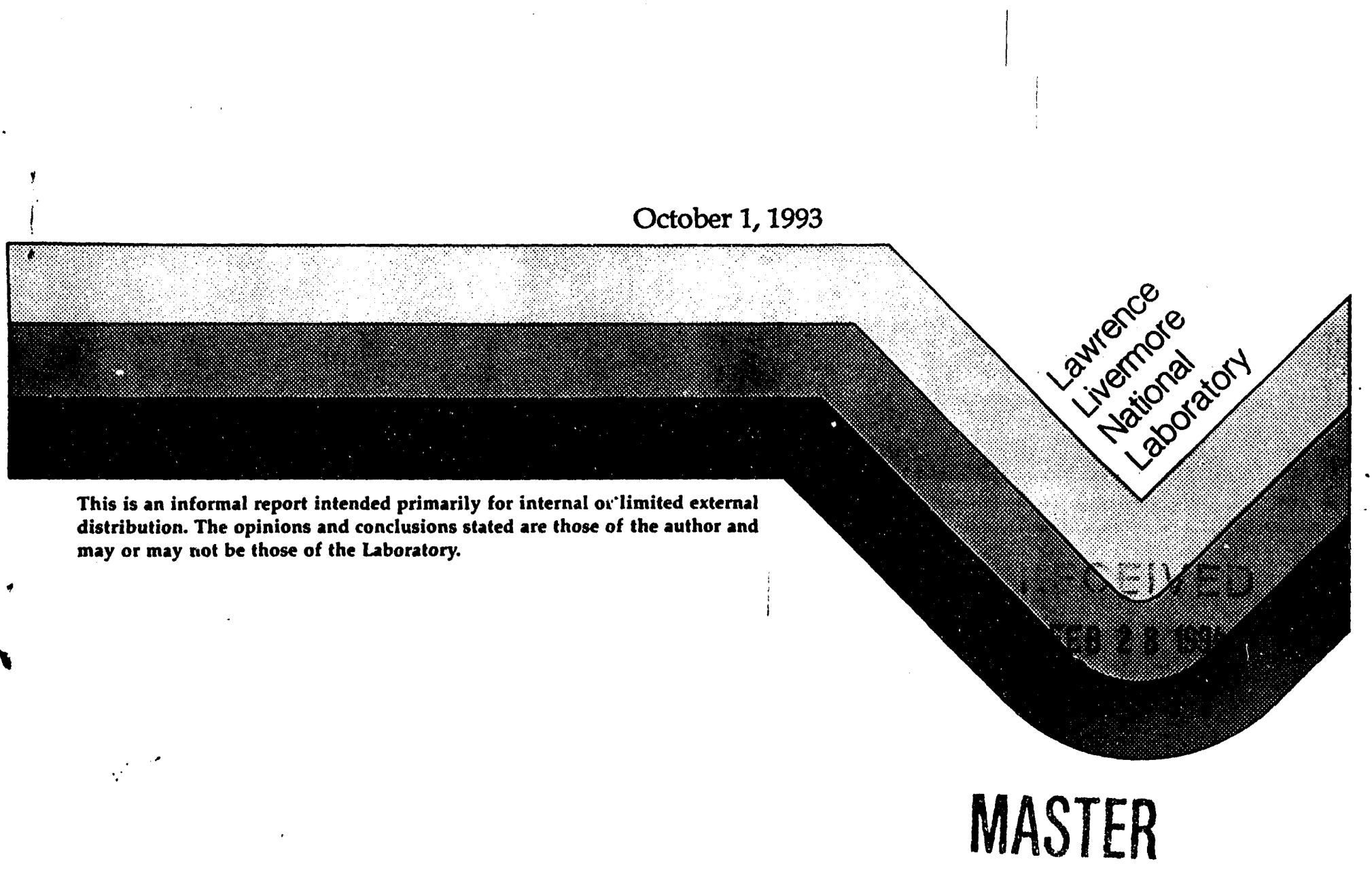

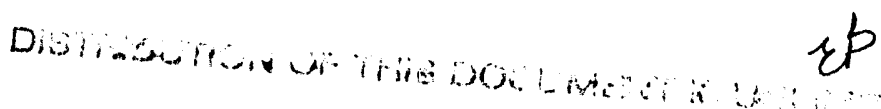




\section{DISCLAIMER}

This document was prepared as an account of work sponsored by an agency of the United States Government. Neither the United States Government nor the University of California nor any of their employees, makes any warranty, express or implied, or assumes any legal liability or responsibility for the accuracy, completeness, or usefuiness of any information, apparstus, product, or process disclosed, or represents that its use would not infringe privately owned rights. Reference herein to any epecific commercial products, process, or service by trade name, trademark, manufacturer, or otherwise, does not necesarily constitute or imply its endorsement, recommendation, or favoring by the United States Government or the University of California. The views and opinions of authors expressed herein do not necessarily state or refiect those of the United States Government or the University of California, and shall not be used for advertising or product endorsement purposes.

This report has been reproduced directly from the best available copy.

Available to DOE and DOE contractors from the Office of Scientific and Technical Information P.O. Box 62, Oak Ridge, TN 37831

Prices available from (615) 576-8401, FTS 626-8401

Available to the public from the National Technical Information Service

U.S. Department of Commerce 5285 Port Royal Rd. Springfield, VA 22161 


\title{
Assessment of Ambient-Temperature, High-Resolution Detectors for Nuclear Safeguards Applications*
}

\author{
Wayne D. Ruhter \\ Nuclear Chemistry Division \\ Lawrence Livermore National Laboratory \\ Livermore, CA 94550 \\ and \\ James H. McQuaid and Anthony Lavietes \\ Electronics Engineering Department \\ Lawrence Livermore National Laboratory \\ Livermore, CA 94550
}

\begin{abstract}
High-resolution, gamma- and $\mathbf{x}$-ray spectrometry are used routinely in nuclear safeguards verification measurements of plutonium and uranium in the field. These measurements are now performed with high-purity germanium (HPGe) detectors, that require cooling to liquid-nitrogen temperatures, thus limiting their utility in field and unattended safeguards measurement applications. Ambient temperature semiconductor detectors may complement HPGe detectors for certain safeguards verification applications. Their potential will be determined by criteria such as their performance, commercial availability, stage of development, and costs. We have conducted an assessment of ambient temperature detectors for safeguards measurement applications with these criteria in mind.
\end{abstract}

\section{Introduction}

Nondestructive assay measurements of nuclear materials for verification purposes is a key component of nuclear safeguards. In most cases, nuclear materials are verified by measuring their neutron and gamma emissions. To detect gamma rays, $\mathrm{NaI}(\mathrm{Tl})$ scintillation and high-purity germanium (HPGe) semiconductor detectors are used. These detectors are well developed and used for many purposes, but are not optimally suited for all safeguards verification tasks. HPGe detectors give excellent energy resolution $(0.5 \%$ at $122 \mathrm{keV})$; however, because of their small bandgap $(0.66 \mathrm{eV})$ they must be cooled to liquid-nitrogen temperatures to reduce thermal noise. This cooling requirement is met either by a bulky dewar, which requires periodic refilling, or a large, electromechanical device with high power requirements. This limits the utility of a HPGe detector in field or unattended applications. NaI scintillation detectors do not require cooling, but their moderate energy resolution (10\% at $122 \mathrm{keV}$ ) is insufficient in most cases for reliable verification measurements.

\footnotetext{
Work performed under the auspices of the U.S. Department of Energy by the Lawrence Livermore National Laboratory under Contract W-7405-Eng-48. This work is supported by the International Safeguards Division in DOE's Office of Arms Control and Nonproliferation.
} 
HPGe detectors have no equals for high-resolution gamma-ray spectrometry and will continue to be the choice for laboratory-based high performance gamma-ray spectrometers. However, there is a continuing desire for an ambient temperature semiconductor detector with the portability and convenience of scintillation detectors, but with a significant improvement in energy resolution. As a result, numerous compound semiconductor materials have been explored, such as $\mathrm{GaAs}, \mathrm{CdTe}$, and $\mathrm{HgI}_{2}$, for use as radiation detectors. Questions remain as to which of these semiconductor materials or whether some new material represents the best option when considering measurement performance, availability, durability, stability, and cost. ${ }^{1}$

\section{Basic Considerations}

In general, all semiconductor detectors depend on charge collection from an active volume of detector material appropriately biased. The volume attainable and the atomic number $(\mathrm{Z})$ of the material are critical parameters in determining the detection efficiency, and generally, both should be large. Another aspect of detector performance is its energy resolution which is determined by the efficiency with which the charge carriers can be collected from the detector's active volume.

The efficiency of charge carrier collection is dependent on the velocity with which the carriers can move and the time they remain free as carriers. In general, the collection efficiency is related to the $E \mu \tau$ product $(E=$ electric field, $\mu=$ carrier mobility, and $\tau=$ lifetime). This product can be thought of as the mean distance that a charge carrier can travel, and this value should be maximized. Therefore, in evaluating any semiconductor detector material, the material's values for these parameters are of primary importance. The size of the electric field, $\mathrm{E}$, that can be applied to a detector is determined by it's resistivity which in turn is related to its bandgap energy and its impurities. Charge carrier (electrons and holes) mobilities and lifetimes are related to the material, how it is made, and the amount and type of impurities in the material. Lifetimes of electrons and holes in germanium are essentially equivalent, while they differ an order of magnitude or more in compound semiconductor materials. Carrier mobilities in compound semiconductors are in general substantially less than in germanium with holes having the least mobility. Cooled germanium detectors typically have $E \mu \tau$ products for the holes and electrons in excess of $10^{4} \mathrm{~cm}$ so that very good charge collection is possible over distances of several centimeters. By contrast, E $\mu \tau$ products obtained for the best compound semiconductor detector materials are on the order of several millimeters for the holes, with most hole E $\mu \tau$ products being much worse. Consequently, collecting holes efficiently from detectors thicker than a few millimeters is difficult. The result is either thin, very low efficiency detectors with excellent energy resolution, or thicker, more efficient detectors with poor energy resolution. Because our need is for high energy resolution and moderately efficient detectors, we need to focus on high-Z semiconductor detectors with higher photon absorption so that they can be made thin. Table 1 gives values for these important parameters for several high-Z, compound semiconductor materials in comparison to germanium. Finally, for detectors to operate at ambient temperature the lower limit for the bandgap energy is $\sim 1.5 \mathrm{eV}$, with an upper limit of $\sim 2.2 \mathrm{eV}$, because the charge carrier mobilities drop rapidly with increasing bandgap energy. 
TABLE 1

Properties of Semiconductor Detectors for Nuclear Radiation Detection

\begin{tabular}{||l|l|l|ll||}
\hline Material & $\begin{array}{l}\text { Maximum } \\
\text { Atomic No. }\end{array}$ & Bandgap (eV) & Hole mobility \\
\hline $\begin{array}{l}\text { Germanium } \\
\text { (Ge) }\end{array}$ & 32 & .67 & $4.2 \times 10^{4}(77 \mathrm{~K})$ \\
\hline $\begin{array}{l}\text { Aluminum } \\
\text { Antimonide } \\
\text { (AlSb) }\end{array}$ & 52 & 1.62 & $350 \quad(300 \mathrm{~K})$ \\
\hline $\begin{array}{l}\text { Cadmium } \\
\text { Telluride } \\
\text { (CdTe) }\end{array}$ & 52 & 1.44 & $80 \quad(300 \mathrm{~K})$ \\
\hline $\begin{array}{l}\text { Cadmium Zinc } \\
\text { Telluride } \\
\text { (CdZnTe) }\end{array}$ & 52 & 1.6 & $280 \quad(300 \mathrm{~K})$ \\
\hline $\begin{array}{l}\text { Gallium } \\
\text { Arsenide } \\
\text { (GaAs) }\end{array}$ & 33 & 2.2 & $40 \quad(300 \mathrm{~K})$ \\
\hline $\begin{array}{l}\text { Mercuric } \\
\text { Iodide } \\
\text { (HgI })\end{array}$ & 80 & 1.45 & $(300 \mathrm{~K})$ \\
\hline
\end{tabular}

3. Zurvey of High-Z Semiconductor Detectors

A. Cadmium Telluride

Cadmium telluride (CdTe) combines a relatively high Z-value (48 and 52) so that the probability of photoelectric absorption per unit length is roughly a factor of 4-5 higher in CdTe than in germanium. To get approximately the same detection efficiency that is obtained with a 15-mm thick, planar HPGe detector typically used for plutonium isotopic measurements would require a CdTe detector thickness of about 4 millimeters. Because of the relatively poor hole collection efficiency, energy resolution performance of CdTe detectors is not comparable with that of HPGe detectors. The best energy resolution reported with a CdTe detector at room temperature has been about $3.5 \mathrm{keV}$ at $122 \mathrm{keV}$. Current CdTe detectors can be operated only up to about 150 volts bias due to poor resistivity and this limits their hole collection efficiency. These detectors are relatively rugged and stable in field use, and can be operated at temperatures up to $30^{\circ} \mathrm{C}$ without excessive noise. 
Most CdTe crystals being used for nuclear detectors are grown by the Travelling Heater Method $(\text { THM })^{2}$. THM grown material is usually doped with chlorine to compensate for vacancies and improve the resistivity. Chlorine doping has been associated with operating instabilities (time dependent decrease in the counting rate and charge collection efficiency). THM crystals are generally of small volume ( $5 \times 5 \times 2 \mathrm{~mm}$ at most) and have low yields of detector grade material thus leading to high detector prices.

Recently, Aurora Technologies Corporation has developed a new, high-pressure Bridgman (HPB) approach to growing detector quality crystals of CdTe and related materials. ${ }^{3}$ HPB crystals are quite large $(10 \mathrm{~cm}$ in diameter and $10 \mathrm{~kg})$, have yields of detector grade material over $70 \%$, and exhibit uniform resistivity without doping. The HPB process can be used to grow high quality crystals of $\mathrm{Cd}_{1-\mathrm{x}} \mathrm{Zn}_{\mathrm{x}}$ Te throughout the alloy composition $(0<\mathrm{x}<1)$ range. Alloying $\mathrm{ZnTe}$ with CdTe increases the bandgap, resulting in much higher resistivities and corresponding lower leakage currents and higher bias voltages than CdTe. We give a comparison of CdTe and $\mathrm{CdZnTe}$ detector performance in the next section of this report.

\section{B. Mercuric Iodide}

Mercuric iodide $\left(\mathrm{HgI}_{2}\right)$ detectors have high resistivities, because of their large bandgap, and correspondingly low leakage currents. Their energy resolution performance is better than CdTe $(2.5 \mathrm{keV}$ at $122 \mathrm{keV})$ except their very poor hole mobility causes considerable low-energy tailing and limits lieir thickness; however, their high atomic number $\left(\mathrm{Z}_{\max }=80\right)$ partially compensates for this. $\mathrm{HgI}_{2}$ detectors are reported to exhibit stability and lifetime problems, related in part to the material's high chemical reactivity and a vapor pressure near atmospheric at room temperatures. The crystal growth process is difficult and not amenable to automation, and the yield of detector-grade material is quite low thus making the detectors relatively expensive.

\section{Gallium Arsenide}

Gallium arsenide (GaAs) has a bandgap sufficiently large to permit ambient temperature operation. The most successful detectors have been made from epitaxial materials in which thin layers are deposited from liquid or vapor phase on a substrate. The thickness of such layers is normally limited to $50-100 \mu \mathrm{m}$, so the active volume and the detection efficiency is quite small. ${ }^{4}$ The lower atomic number of this material also impacts its detection efficiency. Energy resolution performance has not been very good, which is thought to be due to abnormal leakage currents and Johnson noise.

\section{Aluminum Antimonide}

Aluminum antimonide (AlSb) was identified ${ }^{1}$ over fifteen years ago as a compound semiconductor material that had significant potential as a high-resolution, ambient-temperature detector. This was determined on the basis of theoretical calculations that showed its hole mobility to be an order of magnitude larger than CdTe (theoretical hole mobility $=750$ ). Only 
recently has LLNL received funding from the Office of Research and Development to grow these AlSb crystals and investigate their potential as detectors. Recent results show that reasonably large crystals can be grown and the measured hole mobility is on the order of 350 .

4. Results with cadmium-telluride and cadmium-zinc-telluride detectors

We investigated the performance of $\mathrm{CdTe}$ and $\mathrm{CdZnTe}$ gamma-ray detectors from several manufacturers. These detectors were approximately $2 \times 2 \times 2 \mathrm{~mm}$ in size and selected as high resolution, spectroscopy grade devices. We assembled standard, low noise electronic instrumentation to provide a spectroscopy system with the electronic noise contribution to the full width at half maximum (FWHM) being $1 \mathrm{keV}$. CdTe detectors from Radiation Monitoring Devices (RMD), Inc. and Ev Products, and CdZnTe detectors from Aurora Technology Corporation were characterized and compared. Characteristics such as leakage current, bias voltage, FWHM, and FWTM (Full Width at Tenth Maximum) were measured and the results are given in Table 2. Figures 1 and 2 show spectrum measurements of a $\mathrm{Co}^{57}$ source with the Aurora and RMD detectors.

The Aurora CdZnTe detector gave the best performance with $3.3 \mathrm{keV} \mathrm{FWHM}$ at $122 \mathrm{keV}$ and good peak-to-Compton which indicates good charge collection characteristics. The Ev Products detector gave the poorest performance with the FWHM $>6 \mathrm{keV}$ at $122 \mathrm{keV}$. They used a RMD detector packaged with an Ev Products preamp. The specification they gave for this detector was $4 \mathrm{keV}$, however, they do not measure FWHM according to IEEE standards. Their FWHM measurement ignores the low-energy tailing contribution which is caused primarily by poor charge collection.

TABLE 2

Comparison of $8 \mathrm{~cm}^{3}$ detectors ( $1 \mu \mathrm{s}$ time-invariant shaping)

\begin{tabular}{||c|c|c|c|}
\hline & RMD & Ev Products & Aurora \\
\hline Detector bias (volts) & 110 & 150 & 850 \\
\hline Detector leakage (Na) & $>7$ & $>12$ & $<6$ \\
\hline FWHM (keV) & 3.7 & $>6$ & 3.3 \\
\hline FWTM (keV) & 17.5 & $-\cdots$ & 9.0 \\
\hline Cost & $\sim \$ 3,000$ & $\sim \$ 3,000$ & $\sim \$ 300$ \\
\hline
\end{tabular}

The results given above show that the Aurora CdZnTe detector is much superior to the other detectors and at a fraction of the cost. The difference in costs is due to the difference in the way the crystals are made. The HPB method results in larger crystals that give higher yields of detectors. 
The CdZnTe detector has a higher resistivity as indicated by the higher bias voltage and the lowest leakage current. The low leakage current improves the energy resolution performance and the higher bias voltage may improve the hole collection efficiency as indicated by the best FWTM performance. Aurora has indicated that they are continuing to investigate ways to reduce the impurities in the CdZnTe crystals and thus improve the hole lifetimes. This would allow thicker detectors to be made and improve gamma-ray detection efficiency. Aurora also plans to grow crystals with higher zinc content which should increase the resistivity and allow even higher bias voltages. The CdZnTe detector we tested had a molecular $\mathrm{Cd}$ to $\mathrm{Zn}$ ratio of 4 to 1 .

We also tested some alternative signal processing techniques to determine whether the energy resolution performance of these detectors could be improved. As we stated earlier, ambienttemperature, semiconductor materials are known to have poor hole mobility and carrier lifetimes resulting in slow and variable charge collection. This significantly reduces the energy resolution. Time-variant filters are a signal processing technique that may improve the gamma-ray peak shape. A time-variant class of filter is an excellent candidate, because it gives equal weighting to wide distribution of detector signals caused by poor and variable charge collection. This approach to pulse processing, to improve energy resolution, is superior to slow-pulse rejection techniques, because the latter greatly reduces the apparent detection efficiency of the detector.

A time-variant class of filter known as a gated integrator was implemented and tested on the $\mathrm{CdZnTe}$ detector. This resulted in an improved peak shape (more gaussian) and a lower Compton continuum. The FWHM obtained with this configuration was nearly the same as the time-invariant case, but he FWTM was improved significantly.

\section{Summary}

Our assessment of ambient temperature detectors for nuclear safeguards applications shows that $\mathrm{CdZnTe}$ detectors show the greatest potential at this time. From our perspective of applying high-resolution gamma-ray spectrometry to plutonium and uranium isotopic measurements, ambient temperature detectors are limited in their use, because of their current energy resolution performance. Analysis of plutonium gamma-ray spectra for $\mathrm{Pu}$ isotopic information using the

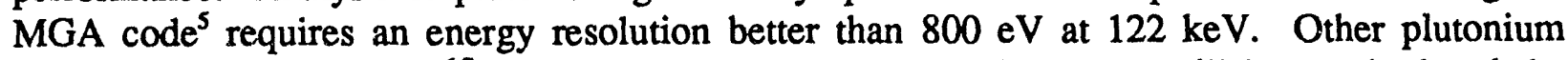
gamma-ray analysis codes ${ }^{6,7}$ may be used, but longer counting times will be required and the accuracy and precision will not be as good as that obtainable with MGA. Analysis of the 100$\mathrm{keV}$ region of uranium gamma-ray spectra for uranium enrichment is not as complex, so the energy resolution requirements are not as severe as with MGA. We estimate that an accurate analysis of the $100-\mathrm{keV}$ region in uranium gamma-ray spectra for uranium enrichment will require an energy resolution of $1.5 \mathrm{keV}$ at $122 \mathrm{keV}$.

Our discussions with Aurora Technologies indicate that there is still a possibility for significant improvement in energy resolution performance of $\mathrm{CdZnTe}$ by increasing the amount of zinc to increase resistivity and by reducing impurity concentrations. We also believe that the higher bias voltages that can be applied to the CdZnTe detectors improve their charge collections properties by collecting the holes faster. We expect that this will allow shorter shaping times with less 
Johnson noise, less variation in charge collection times, and greater improvements in the use of time-variant filters.

We have also discussed with Aurora the possibility of getting a larger CdTe detector. They have a $5 \times 5 \times 5 \mathrm{~mm}$ detector that they are willing to loan us for evaluation. We plan to evaluate its energy resolution and peak-to-Compton performance. We also plan to conduct measurements of nuclear materials with it to determine what isotopic information can be obtained with such a detector. Also, if an aluminum antimonide detector becomes available soon, we will investigate its performance characteristics and evaluate its potential for safeguards applications.

\section{References}

1. G. A. Armantrout, S. P. Swierkowski, J. W. Sherohman, and J. H. Yee, "What Can Be Expected From High-Z Semiconductor Detectors?", IEEE Trans. on Nucl. Sci., Vol. NS-24, No. 1, p. 121 (1977).

2. S. Brelant, M. Elliott, G. Entine, S. Hsu, Revue de Physique Appliquee (Stasbourg France), 141 (1971).

3. J.F. Butler, F.P. Doty, C. Lingren and B. Apotovsky, "Cadmium Zinc Telluride Detectors for Industrial Radiation Measurement," presented at the 2nd Topical Meeting on Industrial Radiation and Radioisotope Measurement Applications, Raleigh, NC, September 8-11, 1992.

4. K. Hesse, W. Gramann and D. Hoppner, Nuclear Instruments and Methods 101, 39 (1972).

5. R. Gunnink, "MGA: A Gamma-Ray Spectrum Analysis Code for Determining Plutonium Isotopic Abundances, Volume 1: Methods and Algorithms", Lawrence Livermore National Laboratory, Livermore, CA, UCRL-LR-103220, Vol. 1 (1990).

6. W. D. Ruhter and W. M. Buckley, "An MS-DOS-Based Program For Analyzing Plutonium Gamma-Ray Spectra, Volume 1 - Data-Analysis Methodology and Software," Lawrence Livermore National Laboratory, Livermore, CA, UCRL-53936, Vol. 1 (1989).

7. T. E. Sampson, G. W. Nelson, and T. A. Kelley, "FRAM: A Versatile Code for Analyzing the Isotopic Composition of Plutonium from Gamma-Ray Pulse Height Spectra," Los Alamos National Laboratory, Los Alamos, NM, LA-11720-MS, (1989). 


$\begin{array}{ll}\text { Detector } & \mathrm{RMD}(2 \times 2 \times 2 \mathrm{~mm}) \\ \text { Shaping Time } & 1 \text { Usec } \\ \text { Bias Voltage } & 110 \text { Volts } \\ \text { Sample Time } & 30 \text { Minutes }\end{array}$

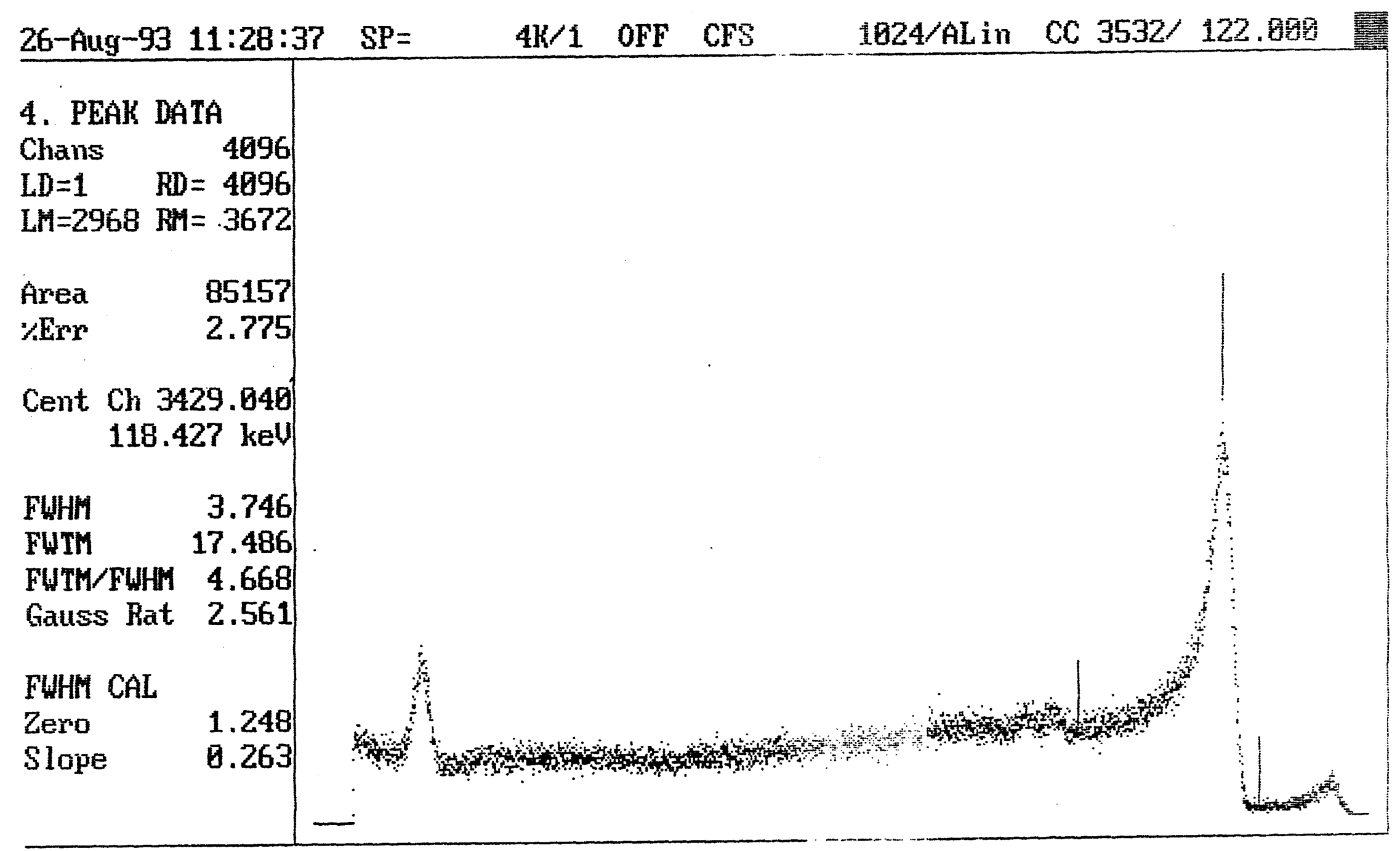

Figure 1. Spectrum of 57 Co taken with a $2 \times 2 \times 2 \mathrm{~mm}$ CdTe detector made by RMD. FWHM at $122 \mathrm{kw}$ was $3.7 \mathrm{keV}$. 


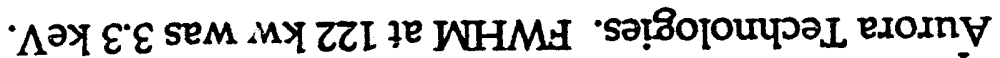

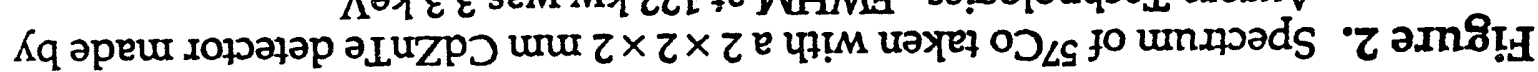

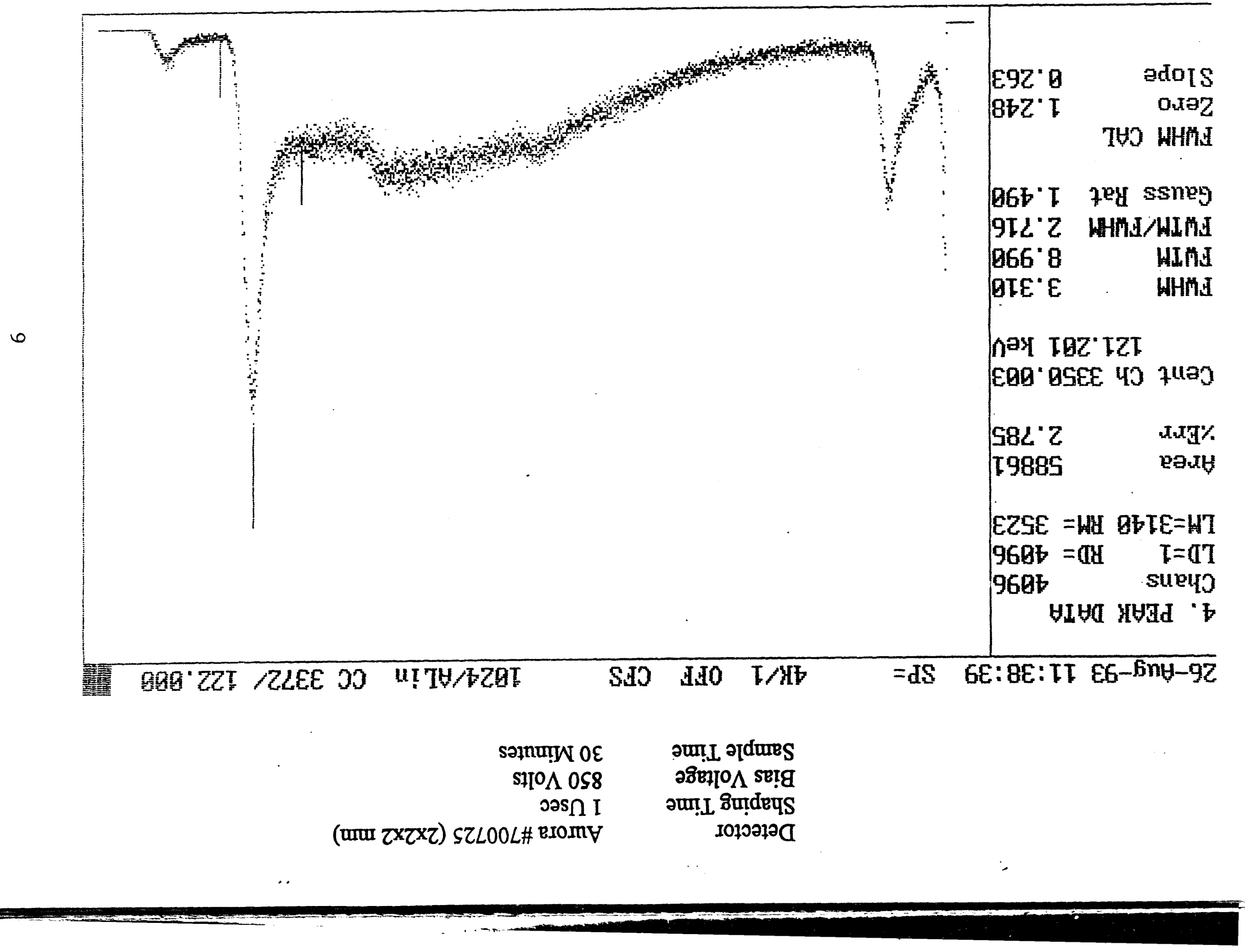



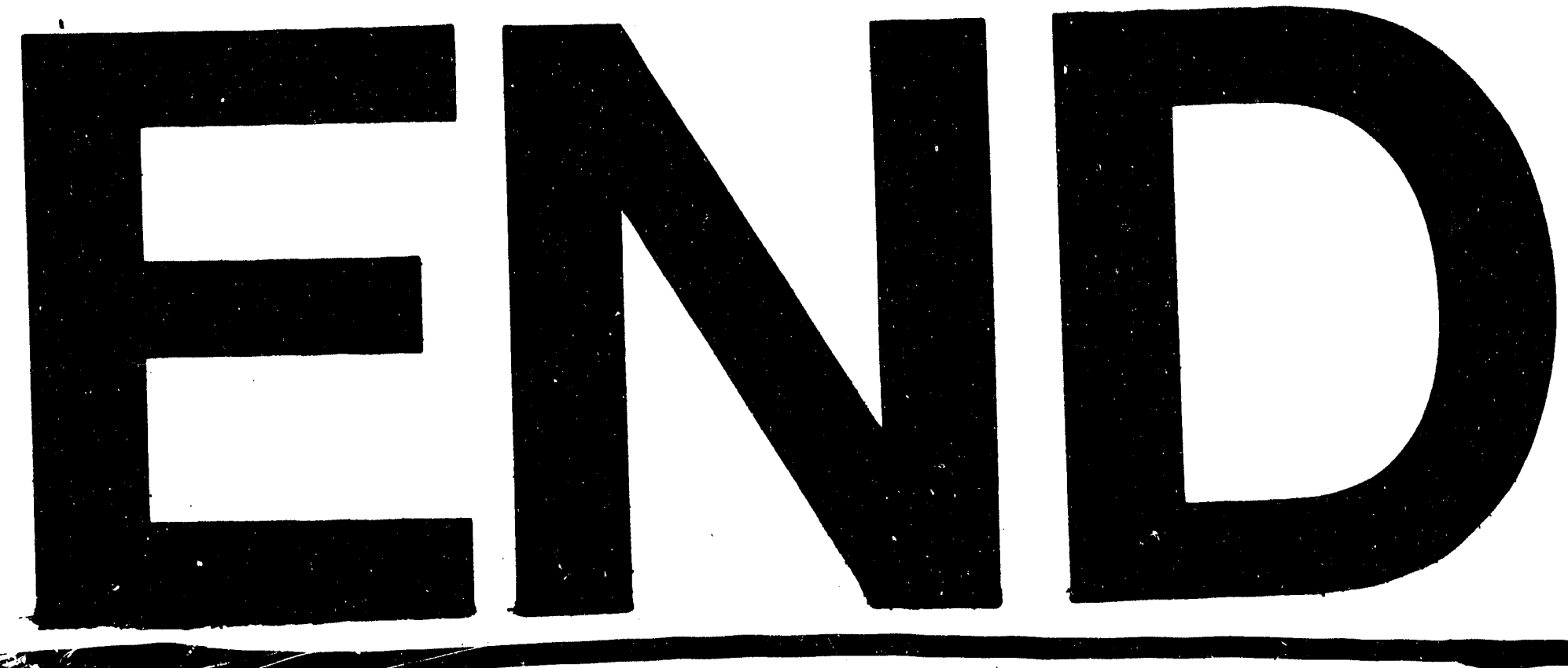

$\infty$

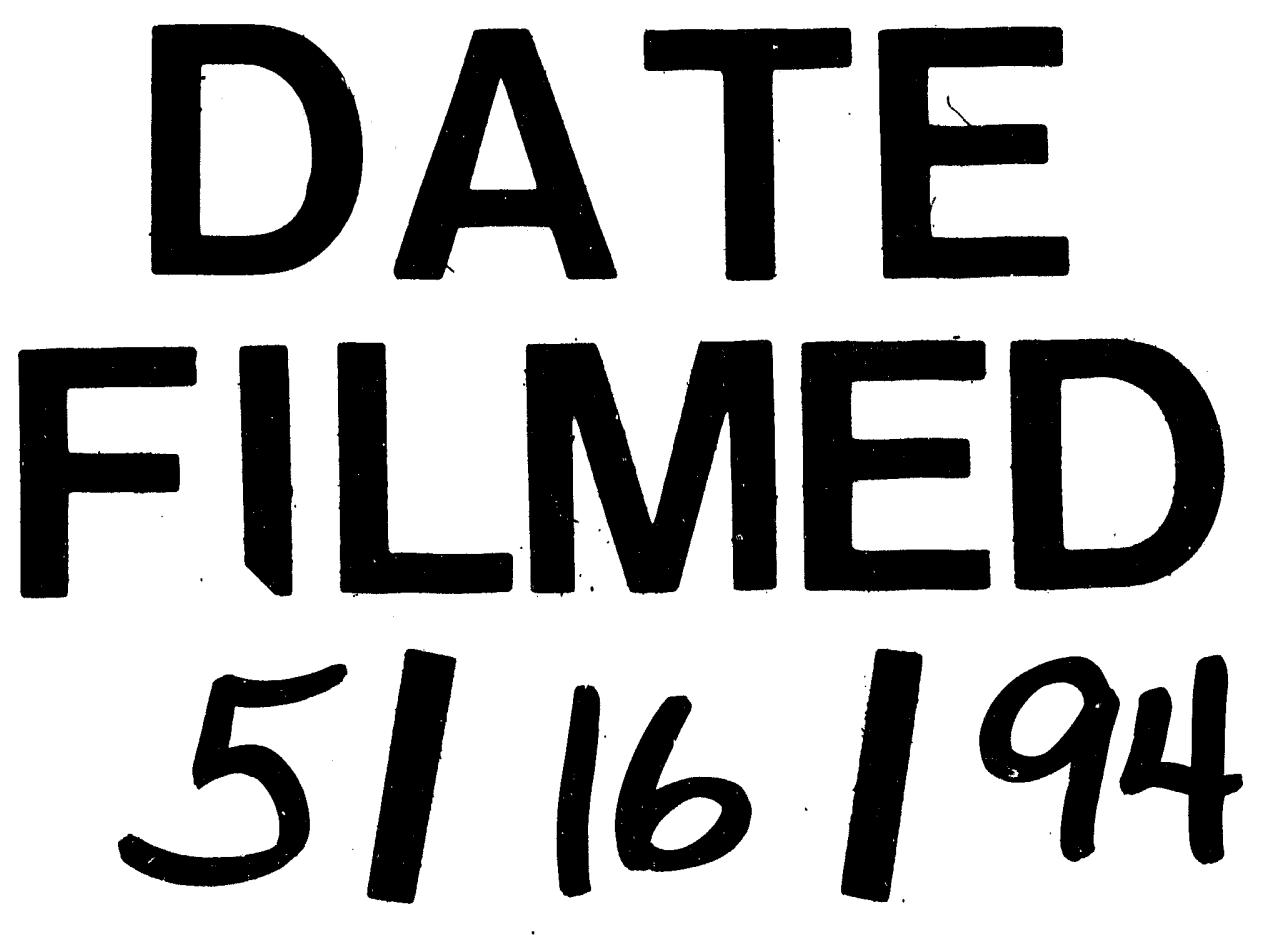

